\title{
APRENDIZAJE TRANSVERSAL A PARTIR DEL ÁREA DE LENGUA Y LITERATURA
}

\author{
CROSS CURRICULAR LEARNING \\ FROM THE AREA OF LANGUAGE AND LITERATURE
}

https://doi.org/10.15304/ie.30.7111

\author{
Joan Mallart Navarra \\ Universidad de Barcelona \\ joan.mallart@ub.edu
}

\section{RESUMEN}

En este artículo se presenta la transversalidad del aprendizaje de competencias comunicativas en ámbitos que no son propiamente lingüísticos, como la comprensión lectora en la resolución de problemas matemáticos o científicos, la elaboración de resúmenes y mapas conceptuales en técnicas de estudio y aprendizaje en las ciencias naturales y sociales, o la realización de debates orales en ciencias sociales o civismo. La idea básica es que con todas las asignaturas se practican las competencias comunicativas. Se puede mejorar el aprendizaje de casi todos los contenidos y competencias que constituyen el campo de las lenguas: el vocabulario, la pronunciación, la comprensión, las técnicas de estudio y la capacidad de expresión oral y escrita. Incluso combinando el lenguaje oral y escrito con otros lenguajes no verbales y gráficos.

Se propone conjugar los contenidos disciplinarios específicos de cualquier área con los del lenguaje utilizado y de la cultura popular subyacente. Se presenta un conjunto de posibilidades temáticas y de actividades didácticas en las que se muestra la potencialidad de este enfoque transversal. Por un lado, medios como la prensa, radio, televisión y nuevas tecnologías se usarán críticamente como fuentes de información. También se sugiere tener presente transversalmente géneros literarios como el teatro la narración, la poesía y la canción para contribuir así a la formación estética.

El contenido del artículo se dirige a todas las etapas educativas, pero si hubiera que destacar alguna sería la Educación Secundaria por la madurez de los estudiantes que les permite leer y comentar textos más complejos, obras extensas, ver películas con un mensaje más definido y escribir textos procedentes de un trabajo colaborativo o de un amplio debate oral entre ellos. En las primeras etapas, casi todo el aprendizaje se basa en el lenguaje comprensivo, expresivo y creativo.

Palabras clave: interdisciplinariedad; temas transversales; enseñanza de la lengua y la literatura; competencias básicas; competencias comunicativas.

\begin{abstract}
This article presents the transversality of the learning of communicative competences in areas that are not strictly linguistic, such as reading comprehension in the resolution of mathematical or scientific problems, the elaboration of summaries and concept maps in study and learning techniques in the natural sciences and social studies, or the realization of oral discussions in the social sciences or civics. The main idea is that communicative competences are practiced with all
\end{abstract}


type of subjects. Almost all the contents and competences that constitute the field of languages can be learned and improved: vocabulary, pronunciation, comprehension, study techniques and oral and written expression skills. Even in combination with other non-verbal and graphic languages. It is proposed to combine the specific disciplinary contents of any area with those of the language used and the underlying popular culture. A set of thematic possibilities and didactic activities is presented, showing the potential of this transversal approach. On the one hand, media such as the press, radio, television and new technologies will be used critically as sources of information. It is also suggested to take into account transversally literary genres such as theater, narration, poetry and song to contribute to the aesthetic formation.

The content of the article is aimed at all stages of education, but if there were one that should be highlighted it would be Secondary Education because of the maturity of the students which allows them to read and comment on more complex texts, extensive works, watch films with a more defined message and write texts that come from collaborative work or extensive oral discussion among them. In the early stages, almost all learning is based on comprehensive, expressive and creative language.

Word keys: interdisciplinarity; cross-curricular themes; teaching language and literature; key competencies; communicative competencies.

\section{VALOR PEDAGÓGICO DEL LENGUAJE: SU PAPEL CENTRAL EN EL CURRÍCULUM}

Todas las tradiciones culturales o religiosas han otorgado al lenguaje un poder creador especial, una naturaleza sagrada que puede transformar el mundo. Al inicio del Génesis, Dios habló. Y cuanto dijo, sucedió. El valor de la palabra es extraordinario. El Evangelio de San Juan empieza afirmando: «Al principio fue la palabra». El nombre de una persona fallecida recientemente es tabú entre algunas culturas, no puede pronunciarse porque hacerlo significaría tener un poder real sobre ella.

Yo soy mi lengua, dirá el poeta palestino Mahmoud Darwich respondiendo a una pregunta sobre su identidad:

«Who am I?

I am my language,

I am an ode, two odes, ten.

This is my language.

I am my language.

I am words' writ...»

Añadirá que desea ser el lugar donde su cuerpo se funde con la eternidad del desierto, así puede convertirse él mismo en sus propias palabras (Darwich, 2003).

Norman Manea, rumano de origen judío, considera a la lengua como su hogar.

Para el escritor, un exiliado por excelencia, la lengua es su placenta. Más que para cualquier otro "extranjero" en su propia tierra, la lengua llega a ser para el escritor no solo una conquista lenta y apasionante, sino la legitimación, el hogar espiritual. A través de la lengua, se siente enraizado y libre, y solo mediante la lengua, hermanado con sus interlocutores potenciales de todas partes. La lengua representa la nacionalidad 
verdadera del escritor, su sentido de pertenencia; la lengua es su casa y su patria [...] Ella continuaría siendo, para mí, el primero y el último refugio, el hogar inmutable de la infancia, el lugar de la supervivencia (Manea, 2008, 23).

En 1908, Unamuno plasmó en un soneto su idea de que la lengua es el espíritu del pueblo que la habla:

«La sangre de mi espíritu es mi lengua,
y mi patria es allí donde resuene
soberano su verbo, que no amengua
su voz por mucho que ambos mundos llene.»

(Unamuno, Soneto XLVII, Rosario de sonetos líricos)

Otra visión de la relación entre lengua y mundo es la conocida frase: «Los límites de mi mundo son los límites de mi lengua» (Wittgenstein), expresión que se puede comprender, pero que en un mundo multilingüe debería carecer de sentido. Es preferible trascender nuestro cerco lingüístico y sentirnos miembros de una humanidad global — ciudadanía planetaria — aunque hable una lengua distinta.

Maragall escribió en un famoso Elogio de la palabra: «No es por el sonido de las palabras que todos los hombres somos hermanos, sino por el espíritu único que las hace brotar diferentes en la variedad misteriosa de la tierra». También reafirmó el valor del lenguaje cuando aconsejaba a los escritores: «Habéis dicho palabras sagradas, no las toquéis...».

Defendiendo la lectura de los clásicos, Lola Josa (2020) propone que «la inteligencia se amplía con la lectura de quienes lograron un dominio del poder de la palabra a través de argumentos, tramas, ensayos o poemas donde transmutaron acontecimientos comunes en universos únicos».

Si aprender es una actitud vital, aprender lenguas lo es más todavía. Para pedagogos que ponen sus objetivos en la transformación, Milani o Freire, el lenguaje posee un elevado poder de liberación personal.

Se ha dicho que todo profesor es profesor de lengua, tópico que por repetido no deja de ser tan cierto como que la lengua ocupa un lugar esencial en el currículum. «Todo profesor es profesor de lengua. Toda lección es una lección de lengua» (Salvador y Camacho, 1991, 98).

Para la profesora sueca Inger Enkvist $(2011,178)$ «todas las materias son lenguas». La lengua es el núcleo del currículum. No porque el profesor de esta asignatura, como todos los de las demás materias, sobrevaloren su campo, sino porque es con y a través del lenguaje, como se presentan los contenidos, se estudian y se aprenden. Incluso se evalúan. No hablamos de situaciones didácticas que utilizan métodos excesivamente verbalistas. También en una metodología de aprendizaje activo por descubrimiento, estudio de casos, proyectos, problemas, etc. el lenguaje tiene un peso considerable. La lengua está presente en todas las materias ya que en ellas se habla, se comprende, se lee, se escribe... Lo explica así Rondal (1980, 145):

La lengua materna se enseña en la escuela en las actividades específicas de lenguaje. Pero es evidente que el aprendizaje de la lengua se hace de una manera permanente a través de las 
otras actividades escolares. Las actividades físicas y deportivas implican una comunicación oral entre los participantes. Las actividades matemáticas utilizan un simbolismo especial, pero el lenguaje común sirve para formular problemas y dar explicaciones. El maestro enseña, pues, la lengua materna en todo momento; por lo tanto, esta enseñanza no es intelectual [...]

La lengua materna es el vehículo de todos los aprendizajes y el medio de integración en la comunidad escolar. Es importante poner en cuestión la calidad de los intercambios verbales entre alumnos y maestros en el marco de la clase. En gran medida, estos intercambios constituyen el proceso de la educación.

Abundando en el mismo mensaje, los poetas dirán que «el lenguaje no tiene meramente una función comunicativa: sirve también para construir lo que no existe, para sugerir lo que no es conocido, lo que es solo pensado o soñado» (Carles Riba). Añadiendo Pedro Salinas, en la misma línea de Maragall -a quien admiraba-, que el ser humano es inseparable de su lenguaje... «La palabra es espíritu, no materia, y el lenguaje, en su función más trascendental, no es técnica de comunicación: es liberación del pobre, es reconocimiento y posesión de su alma, de su ser» (Salinas, 1967).

Por eso, la lengua en el centro del currículum no debería ser solo una cuestión instrumental o técnica. Además del cerebro, está el corazón y el homo sapiens es también homo loquens. Poner la enseñanza de la lengua, con su cultura y especialmente su literatura, en el núcleo del currículum significa darle su función fundamental interpretativa y crítica de nosotros mismos y del mundo, junto a la función creativa, expresiva del pensamiento. La apertura y la implicación en el mundo que nos rodea, para que nada humano nos resulte extraño, como querían Terencio y los clásicos, a través de la revalorización de estas funciones comunicativas esenciales nos puede devolver el Humanismo más necesario que nunca en este momento.

El corazón de un currículum es la lengua y el corazón de una lengua es su cultura popular. De ahí que en los primeros cursos de educación infantil, e incluso en primaria, tengan tanta importancia las manifestaciones lingüísticas de la literatura popular, como retahílas, canciones, cuentos, refranes, adivinanzas... que permitirán conservar el alma de una lengua y su cultura.

\section{FUNDAMENTOS DE LA TRANSVERSALIDAD EN EL APRENDIZAJE}

La transversalidad en el currículum español apareció oficialmente con la reforma de los años 90 del siglo pasado y fue una manera de innovar con interesantes propuestas didácticas. Ante la estricta división de las asignaturas tradicionales, se iniciaban agrupaciones de contenidos que pertenecían a distintas áreas, de manera permeable y permitían un trabajo interdisciplinario. También presentaban como ventaja la de ofrecer una mayor relación con la vida real. Los temas transversales responden a problemas actuales de gran relevancia y contribuyen poderosamente a una educación integral mediante la consideración de los valores propios de cada tema, valores que se encuentran en distintas disciplinas educativas. Al respecto, nos dirá Rodríguez Rojo que estos temas: 
Suponen una respuesta a la problemática social en boga. Contienen valores universalmente consensuados; se prestan a la interdisciplinariedad pues atraviesan las vías del conocimiento, no se identifican con las áreas, ni mucho menos con las asignaturas, y promueven la convivencia en democracia. (Rodríguez Rojo, 1996, 97).

La característica principal de los contenidos que denominamos temas transversales es la de desbordar una sola área. Su función consiste en conectar y articular conocimiento y formación, estableciendo conexiones entre saber, hacer, ser y convivir. Rosales sugiere que estos contenidos:

Por su relevancia formativa no pueden confinarse en el ámbito de una determinada disciplina o área curricular, sino que deben ser objeto de tratamiento en todas o en una pluralidad de ellas. Se trata pues, de contenidos que se van a desarrollar "a través de" la mayoría de actividades que el alumno realiza en el centro escolar (Rosales, 2015, 144).

Debemos recordar, con Yus $(1996,36-37)$ que los temas transversales no querían ser nuevas asignaturas con las que cargar más todavía el currículum, sino núcleos de interés que podían hacer de puente entre una cultura popular real y la cultura escolar, aproximando una a la otra. Según este autor, no se trataría de contenidos separados ni paralelos a las áreas, ni mucho menos inconexos entre sí. La idea que ha de unir la transversalidad con la disciplinariedad debe ser un espíritu humanizador que aproxime las diferentes facetas de la educación integral. De las críticas constructivas de Yus (1996) y Rosales $(2015,2019)$ principalmente, entre otros (Palos, 1998; González Lucini, 1994; Rodríguez Rojo, 1996) podemos extraer algunas ventajas de este enfoque transdisciplinario. Las dificultades, si se lleva a la práctica de una manera precipitada o incorrecta, serían una mayor carga para el alumno por la posibilidad de aumentar los contenidos, y la fragmentación si no se enlazan bien los temas. Las ventajas son muy superiores:

Se explicitan valores que contribuyen a una educación humanista integral.

Son temas de interés social.

Motivación intrínseca por su mayor relación con la vida real.

Interrelación de contenidos entre áreas.

Calidad de la formación gracias a la colaboración del equipo docente que programa conjuntamente.

Uso de metodologías más activas, de investigación en grandes núcleos, casos, problemas o proyectos, atendiendo a la diversidad.

Didáctica menos rutinaria y que estimula la creatividad.

No es difícil aplicar estas importantes ventajas a una transversalidad a partir de contenidos culturales de lengua y literatura. De hecho, la lengua no aparece en casi ninguna relación de temas transversales, pero atendiendo a la conceptualización que presentamos, no parece que haya inconveniente en considerar un amplio conjunto de contenidos relacionados con la 
comunicación en los que la lengua tendría un papel preponderante, aunque no único. No se propone un nuevo tema transversal, sino algo mucho más ambicioso: que la lengua y la literatura se conviertan en un eje que pueda aglutinar a la mayoría de los temas, puesto que en todos ellos va a ser necesaria su contribución. A la vez, trabajando contenidos de otras áreas, se profundiza en aspectos comunicativos, lingüísticos o literarios. Sin limitarse al currículum formal, se aprenden competencias lingüísticas en espacios como la vida social de la escuela, en la biblioteca, en actividades recreativas y en los medios de comunicación.

La metodología dominante en el momento en que apareció el interés por la transversalidad era el constructivismo. Hoy, los documentos curriculares reflejan un enfoque por competencias generalizado en el panorama educativo occidental. No hay duda de que esta visión competencial incluye el trabajo interdisciplinario a partir de la lengua.

\section{EL CURRÍCULUM POR COMPETENCIAS COMO FACILITADOR DEL APRENDIZAJE TRANSVERSAL}

Ante la presencia de las competencias en el currículum, después de revisar aportaciones internacionales, definimos competencia como:

la capacidad de utilizar conocimientos y habilidades, de manera transversal e interactiva, en contextos y situaciones que requieran la intervención de conocimientos vinculados a diferentes saberes, lo que implica la comprensión, reflexión y discernimiento teniendo en cuenta la dimensión social de cada situación» (Mallart, 2011, 251).

El enfoque por competencias supone un saber práctico, contextualizado y transversal.

Entre las competencias básicas del currículum primario, secundario, o universitario, se definen unas competencias disciplinarias más relacionadas con los contenidos de cada asignatura, y otras competencias que se suelen denominar transversales porque no están adscritas a una sola materia. Entre estas últimas destacan unas competencias cognitivas (responder y formular preguntas pertinentes; elaborar mapas conceptuales; resolver problemas; inventar y plantear nuevos problemas), relacionales (colaborar en el equipo; dirigir un grupo), organizacionales (planificar) y sobre todo comunicativas (comprender mensajes orales o escritos, tomar notas; realizar presentaciones orales, escritas o gráficas, con recursos digitales o analógicos). 
Cuadro 1. Trabajo integrado de inteligencias múltiples, competencias y ejes transversales.

\begin{tabular}{|c|c|c|c|}
\hline $\begin{array}{l}\text { Dimensiones de la } \\
\text { educación integral }\end{array}$ & $\begin{array}{l}\text { Inteligencias múltiples } \\
\text { (Gardner) }\end{array}$ & Competencias básicas & Ejes o temas transversales \\
\hline $\begin{array}{l}\text { Dimensión intelectual } \\
\text { o cognitiva }\end{array}$ & Inteligencia lingüística & $\begin{array}{l}\text { Competencia comunicativa } \\
\text { lingüística y audiovisual }\end{array}$ & $\begin{array}{l}\text { Educación en los Medios de } \\
\text { Comunicación }\end{array}$ \\
\hline \multirow{4}{*}{$\begin{array}{l}\text { Conocimientos, ideas, } \\
\text { racionalidad, verdad, } \\
\text { creatividad }\end{array}$} & & Competencia matemática & \\
\hline & $\begin{array}{l}\text { Inteligencia lógico- } \\
\text { matemática }\end{array}$ & $\begin{array}{l}\text { Tratamiento de la información } \\
\text { y competencia digital }\end{array}$ & -Educación Comunicativa- \\
\hline & & $\begin{array}{l}\text { Competencia para aprender a } \\
\text { aprender }\end{array}$ & \\
\hline & Inteligencia naturalista & $\begin{array}{l}\text { Conocimiento e interacción } \\
\text { con el mundo físico }\end{array}$ & $\begin{array}{l}\text { Educación Ambiental } \\
\text { (Ecoformación) }\end{array}$ \\
\hline $\begin{array}{l}\text { Dimensión física } \\
\text { Cuerpo, percepción } \\
\text { sensorial, motricidad }\end{array}$ & $\begin{array}{l}\text { Inteligencia cinético- } \\
\text { corporal }\end{array}$ & $\begin{array}{l}\text { Conocimiento e interacción } \\
\text { con el mundo físico }\end{array}$ & \multirow[t]{2}{*}{ Educación para la Salud } \\
\hline Dimensión personal & $\begin{array}{l}\text { Inteligencia } \\
\text { intrapersonal }\end{array}$ & Competencia emocional & \\
\hline Dimensión social & \multirow[t]{4}{*}{$\begin{array}{l}\text { Inteligencia } \\
\text { interpersonal }\end{array}$} & \multirow[t]{4}{*}{$\begin{array}{l}\text { Competencia social y } \\
\text { ciudadana }\end{array}$} & $\begin{array}{l}\text { Educación para la } \\
\text { Convivencia, los Derechos } \\
\text { Humanos y la Paz. } \\
\text { Resolución de Conflictos }\end{array}$ \\
\hline \multirow{2}{*}{$\begin{array}{l}\text { Cultura, politica, } \\
\text { economía, relaciones } \\
\text { interpersonales }\end{array}$} & & & Educación para la Igualdad \\
\hline & & & $\begin{array}{l}\text { Educación para el } \\
\text { Consumo responsable }\end{array}$ \\
\hline $\begin{array}{l}\text { Valores sociales: } \\
\text { justicia, igualdad }\end{array}$ & & & Educación Vial \\
\hline Dimensión moral & & \multirow{3}{*}{$\begin{array}{l}\text { Competencia para la } \\
\text { autonomía e iniciativa } \\
\text { personal }\end{array}$} & \multirow{3}{*}{ Educación Ética o Moral } \\
\hline Valores personales & & & \\
\hline $\begin{array}{l}\text { Dimensión volitiva } \\
\text { Voluntad, deseos, } \\
\text { motivación, esfuerzo, } \\
\text { autodominio }\end{array}$ & & & \\
\hline $\begin{array}{l}\text { Dimensión } \\
\text { transcendente }\end{array}$ & Inteligencia existencial & Competencia espiritual & $\begin{array}{l}\text { Educación de la } \\
\text { Interioridad }\end{array}$ \\
\hline $\begin{array}{l}\text { Dimensión estética } \\
\text { Arte, creatividad }\end{array}$ & $\begin{array}{l}\text { Inteligencia musical } \\
\text { Inteligencia espacial }\end{array}$ & $\begin{array}{l}\text { Competencia artística y } \\
\text { cultural }\end{array}$ & Educación Artística \\
\hline
\end{tabular}

Esta transversalidad en las competencias que se deben adquirir exige la participación de todo el equipo de profesorado, coordinado para que las enseñanzas no aparezcan inconexas entre sí y gracias al trabajo interdisciplinario, establezcan mayor relación del alumnado con la vida real. De esta manera será más fácil que cada estudiante sea más autónomo, comprometido, trabaje con iniciativa personal y esté implicado en su propio aprendizaje con una motivación 
intrínseca. Combinando las competencias que demanda el currículum con las inteligencias múltiples de Gardner y, a su vez, con los temas transversales, presentamos un cuadro general que comienza con la competencia comunicativa (Cuadro 1).

Para los aprendizajes transversales lingüísticos, se propone en primer lugar la competencia comunicativa, que sobrepasa a una mera competencia lingüística o gramatical.

La competencia comunicativa es la capacidad de comprender y formular enunciados en el contexto concreto. Regula cómo se ha de usar el lenguaje para ser apropiado a la situación y al interlocutor. Se dirige al uso, es práctica. Supone la competencia lingüística y va más allá. Porque aprender una lengua no consiste solo en aprender su gramática, sino también en cómo llevar a cabo las intenciones propias usando esta gramática adecuadamente. Las competencias que componen la competencia comunicativa son:

a) Competencia lingüistica: Conocimiento del sistema de la lengua, de la estructura de las frases y de la pertinencia de las formas lingüísticas. Habilidad para interpretar y aplicar las reglas del código: de pronunciación y entonación (competencia fonológica), habilidad gráfica (competencia grafológica), competencia léxica y morfosintáctica.

b) Competencia textual: Habilidad para reconocer y usar diferentes tipos de discurso, conectar frases o realizaciones lingüísticas de un gran conjunto temático. Se reconoce y acierta en la elección del tipo de texto: descripción, narración, diálogo, instrucciones, argumentación, exposición, comentario, epistolar, periodístico, poesía... En ciencias naturales y sociales se trabajan y elaboran textos literarios y científicos. En tecnología predominan textos funcionales.

c) Competencia sociolingüística: Habilidad para comprender y usar el lenguaje apropiado a cada contexto, adaptándose a los roles de los participantes, la situación, las funciones de la comunicación y el propósito de la interacción o intenciones comunicativas. Se puede practicar en cualquier materia, teniendo en cuenta los roles de alumno y profesor.

d) Competencia estratégica: Capacidad para definir, corregir, matizar, o realizar ajustes en el curso de la situación comunicativa para compensar el imperfecto o incompleto conocimiento de las reglas lingüísticas. Aunque se practica en todas las asignaturas cuando no se comprende y se piden explicaciones, es muy pertinente en clase de idioma moderno.

e) Competencia cultural (o intercultural): Conocimiento del contexto social y cultural en el que se utiliza una lengua. Corresponde al conocimiento del mundo, familiaridad con elementos externos de la lengua, como son los espacios de participación y de intervención, la distribución del tiempo, las demostraciones de familiaridad o distancia, el conjunto de conocimientos sobre la vida cotidiana, valores y actitudes propios de una cultura (Lladó y Llobera, 1999, 67). Es una competencia muy necesaria en el aprendizaje de una lengua que no sea la materna, y se puede adquirir de manera transversal en espacios menos formales y en la enseñanza formal de cualquier otra disciplina distinta de la lengua. Sin olvidar la cultura popular con sus fiestas, tradiciones, cancionero, refranero o incluso enigmística. 
También se han llamado tradicionalmente competencias comunicativas a las cuatro habilidades de comprender, hablar, leer y escribir. En ellas encontraremos actividades lingüísticas que se pueden desarrollar en cualquier área curricular o tema transversal.

Cuadro 2. Contenidos transversales de las habilidades lingüísticas.

\begin{tabular}{|c|c|c|}
\hline & Habilidades receptivas & Habilidades productivas \\
\hline$\frac{\mathscr{0}}{\tilde{\pi}}$ & $\begin{array}{l}\text { Comprensión auditiva } \\
\text {-Atención } \\
\text {-Memoria } \\
\text {-Discriminación aditiva } \\
\text {-Inferencia } \\
\text {-Anticipación } \\
\text {-Interpretación }\end{array}$ & $\begin{array}{l}\text { Expresión oral (hablar) } \\
\text {-Análisis de la situación, audiencia, proxémica } \\
\text {-Planificación del mensaje } \\
\text {-Exposición, entonación, pronunciación } \\
\text {-Aspectos no verbales }\end{array}$ \\
\hline 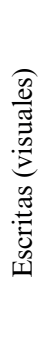 & $\begin{array}{l}\text { Comprensión lectora (leer) } \\
\text {-Vocabulario } \\
\text {-Inferencia } \\
\text {-Anticipación } \\
\text {-Interpretación } \\
\text {-Localización de la idea principal } \\
\text {-Síntesis, resumen }\end{array}$ & $\begin{array}{l}\text { Expresión escrita (escribir) } \\
\text {-Tipo de texto, género } \\
\text {-Grado de formalidad } \\
\text {-Coherencia, cohesión } \\
\text {-Planificación } \\
\text {-Redacción } \\
\text {-Revisión } \\
\text {-Publicación }\end{array}$ \\
\hline
\end{tabular}

Como prueba de que estas habilidades o competencias se hallan en la mayoría de las áreas del currículum, se presenta a continuación una muestra de su transversalidad.

a) Vocabulario. La competencia léxica es necesaria en cualquier área, todas tienen su vocabulario específico, una terminología propia que se debe comprender, definir y utilizar con precisión.

b) Interacción oral (Comprensión auditiva y expresión oral). Se realizan constantemente juegos orales, diálogos, debates, juegos de rol, dramatización, teatro, narración en voz alta de chistes o cuentos. Medios de comunicación como la radio y la televisión deben tener un papel en varias asignaturas para el conocimiento de la actualidad y del entorno social y natural.

c) Comprensión lectora. En todas las áreas se practica la lectura comprensiva y crítica de textos de tipología diversa: literarios, científicos, históricos, de los medios de comunicación. Aquí la biblioteca cobra una importancia decisiva, se pueden desarrollar clubs de lectura y sirve para obtener información sobre cualquier tema. La lectura es una de las principales técnicas de estudio. Junto con los libros, hoy Internet se usa también para localizar y elaborar conocimiento. La biblioteca no entra en competición con los medios digitales, se complementan mutuamente.

d) Expresión escrita. Además de la expresión creativa, también se utilizará con una función instrumental elaborando resúmenes, mapas conceptuales, cuadros sinópticos... Y utilizando también las tecnologías de la información con sus potentes herramientas como 
diccionarios y correctores. El uso de la correspondencia — convencional y electrónica-con personas de fuera de la escuela amplía el uso y consolida esta competencia.

Las aulas están decoradas, llenas de palabras, frases, poemas, textos en carteles ilustrados. La lengua es omnipresente. En cualquier materia puede haber un club de escritura, un taller y un concurso literario o científico sobre su temática propia.

\section{PROYECTOS Y ACTIVIDADES QUE RELACIONAN LA LENGUA Y LA LITERATURA CON OTRAS ÁREAS}

Como ejemplos de transversalidad, sugerimos tan solo algunas propuestas de actividades, partiendo de la lengua o la literatura. A través de ella se aprenderán competencias y contenidos próximos a otras disciplinas, en modo alguno ajenos al lenguaje mismo o a su expresión literaria. Como es natural, son solamente unos ejemplos sin pretensión de exhaustividad. Lo importante es lo que puedan plantear los profesores desde su trabajo en equipo, su conocimiento del entorno físico y cultural así como desde los intereses de su alumnado.

Con estas premisas, con la creatividad y el trabajo en equipo, la motivación, la consecución de las competencias va a ser de mucha mayor calidad. Para ello harán falta metodologías globalizadoras como estudios de casos, problemas, proyectos, créditos de síntesis, grandes unidades didácticas con participación de materias variadas.

\subsection{Lengua y literatura, lenguas de las comunidades e idiomas modernos}

Las actividades lingüísticas tienen la máxima importancia en el desarrollo de la competencia comunicativa. Al ser una competencia transversal, la consecución de sus objetivos dependerá del tratamiento que se haga en conjunto. A la vez, las actividades relacionadas con esta competencia favorecerán la mejora de las competencias metodológicas.

Para la enseñanza de lenguas modernas hay un método llamado Aprendizaje Integrado de Contenidos en Lengua Extranjera (AICLE) que resulta ser un modelo de transversalidad porque a través del lenguaje se enseñan todas las demás disciplinas. Y además, se aprende la lengua. Este modelo es el que se propone aquí, incluso para la lengua materna.

El cuento y la narración en general, la poesía y el teatro utilizados en cualquier área contribuyen al desarrollo de la competencia literaria y la competencia textual, entre otras. Y pueden motivar, siempre que la escuela y el profesorado vivan la literatura. En una excursión podemos encontrar poemas grabados en mosaicos o piedras del bosque. También podemos leer en el mismo lugar descripciones literarias de autores.

\subsection{Matemáticas}

Puede parecer que esta es la materia menos proclive a tratar de desarrollar competencias comunicativas. Pero la Matemática es también un lenguaje y nos sorprenderían las relaciones que se pueden establecer con la lengua. En Matemáticas y Ciencias se necesita la comprensión verbal y también la expresión cualitativa y cuantitativa con exactitud y precisión, sin ambigüedades. 
Para deducir la importancia y necesidad del lenguaje en el área de Matemáticas tendremos en cuenta las competencias que se deben desarrollar. Según Niss (1999) serán las siguientes: 1) Pensar y razonar; 2) Argumentar; 3) Comunicar; 4) Modelar (traducir la realidad a una estructura matemática); 5) Plantear y resolver problemas; 6) Representar; 7) Utilizar el lenguaje y operaciones simbólicas, formales y técnicas; 8) Utilizar ayudas y herramientas. De las ocho enunciadas, al menos cinco requieren componentes relacionado con el lenguaje, especialmente argumentar, comunicar y utilizar el lenguaje.

Las matemáticas contribuyen al desarrollo de la competencia comunicativa aportando un lenguaje específico, necesario para todas las ciencias y para la resolución de problemas cotidianos. En el aprendizaje matemático, el uso de la lengua, oral o escrito, junto con un vocabulario muy preciso, es necesario para comprender los enunciados de los problemas, describir conceptos y procesos, expresar razonamientos, argumentaciones y pruebas, así como para comunicar, discutir, comparar y validar los datos obtenidos.

Entender un problema es necesario para encontrar la solución. Para ello hace falta comprender un vocabulario general y también términos más específicos. En el primer caso, palabras como: 'todos, ninguno, más, menos, repartir, añadir, sustraer'... Y expresiones como: 'ya que, por consiguiente, de esto se desprende que’...

Un ejercicio interesante consiste en verbalizar el proceso de resolución de un problema, sin resolverlo numéricamente. Plantear y explicar el algoritmo, indicando y justificando las operaciones que se harían para llegar a la respuesta. Igualmente útil es inventar problemas, redactando sus enunciados.

Los números y cantidades aparecen en poemas y canciones, en juegos de lápiz y papel (hundir la flota), en el dominó, cartas, dados. Al jugar se tiene que interactuar hablando, también se suele contar, como en el parchís y llevar la cuenta de la puntuación de cada jugador. Se pueden proponer enigmas, adivinanzas matemáticas, otra forma de problemas

Cuatro perros en un cuarto, cada perro en un rincón, cada perro ve tres perros. Adivina cuántos perros son (cuatro).

Soy más de uno, sin llegar a tres, y llego a cuatro cuando dos me des (dos).

Dos padres y dos hijos se sentaron a comer huevos. Cada uno se comió uno y en total se comieron tres huevos. ¿Cómo puede ser?

La poesía requiere contar las sílabas de un verso para que todos sean iguales, y también contar el número de versos para componer las estrofas: sílabas, versos, hemistiquios y estrofas tienen un número. Se han escrito poemas al número pi, juego verbal mnemotécnico para recordar sus decimales.

Soy П, lema y razón ingeniosa de hombre sabio, que serie preciosa valorando enunció magistral... (Rafael Nieto)

Se pueden encontrar en la literatura obras o fragmentos de tema matemático. Como: 
Shakespeare, Soneto XVIII (Sé la musa que hace diez, diez veces...)

Verne, 20000 leguas de viaje submarino

Alberti, A la divina proporción

Neruda, Oda a los números

Unamuno, La tabla de multiplicar

O novelas matemáticas y obras de divulgación:

Malba Tahan, El hombre que calculaba

Han Magnus Enzesberger, El diablo de los números

Denis Guedj, El teorema del loro

John Allen Paulus, El hombre anumérico

Para celebrar el Día del Número Pi, el 14 de marzo, se pueden realizar proyectos didácticos transversales, concursos de problemas, concursos de poesía matemática, o ver películas (Donald en el país de las Matemáticas, La habitación de Fermat...).

\subsection{Ciencias Naturales, Educación Ambiental y Educación para la Salud}

Las ciencias aportan al aprendizaje del lenguaje un registro propio, el lenguaje de la ciencia, indisociable del propio conocimiento científico. El registro culto científico se concreta en formas específicas de describir los hechos y fenómenos, explicarlos, exponerlos, justificarlos y argumentarlos, así como definirlos y clasificarlos. La complejidad de los hechos y fenómenos estudiados requiere el complemento de otros lenguajes comunicativos como gráficos y multimedia.

La actividad científica sigue un proceso según unas etapas que suelen ser: a) observar, comprender y plantear el problema, b) establecer hipótesis, c) obtención de datos, d) analizar los datos y e) interpretar los resultados y sacar conclusiones. Este proceso de razonar con lógica y rigor también se puede realizar en clase de lengua:

Si el profesor de lengua, durante la clase de gramática, habitúa a los alumnos a observar una frase, a modificar sus partes y a razonar sobre los cambios obtenidos, realiza una actividad educativa que en cierto modo saca partido de los mismos procesos mentales de la enseñanza de las ciencias, de acuerdo con los cuales los niños se acostumbran a observar un fenómeno y a ir aislando en él sus diferentes parámetros para tratar de descubrir aquéllos cuya influencia es decisiva (Ceccarelli, 1977, 187).

Enkvist encuentra también muchas conexiones entre el lenguaje y las ciencias, Citando a Lemke (1997) afirma que estudiar ciencias naturales es como estudiar lengua porque constata que:

Los alumnos usan la lengua para muchos propósitos como observar, describir, comparar, clasificar, analizar, discutir, formular hipótesis, teorizar, cuestionar, retar, argumentar, diseñar experimentos, llevar a cabo procedimientos, juzgar, evaluar, decidir, concluir, generalizar, divulgar, escribir y, a su vez, enseñar (Enkvist, 2011, 195).

Hay diferentes tipos de textos científicos para debatir y comunicar datos e ideas: descripción, explicación, definición, clasificación, exposición, justificación y argumentación. Se 
recomienda la lectura crítica de textos con contenido científico, divulgativos, sobre descubrimientos, biografías de inventores. Se leen y se escriben textos que dan cuenta de los resultados de los experimentos. Se pueden encontrar elementos científicos en novelas de ciencia-ficción.

Podemos proponer descripciones escritas de animales, plantas o minerales sin decir a qué corresponden. Al leerlas en voz alta, los compañeros deberán descubrir a qué corresponde cada descripción. Tenemos poemas de plantas: A un olmo seco (Machado), El ciprés de Silos (Gerardo Diego)... Y también fábulas de animales. Las biografías de inventores son textos de interés que comparten aspectos lingüísticos con aspectos históricos y científicos. Muchas vocaciones para la ciencia han nacido así.

Durante las excursiones o paseos por la naturaleza se aprovechan conversaciones sobre vivencias de naturalistas. Si se recogen materiales, se pueden realizar exposiciones por los propios alumnos, escribiendo rótulos precisos con información breve. Investigando y discutiendo se deshacen mitos y conceptos erróneos: salir el sol, dar calor, cerebro gris, cinco sentidos...

\subsubsection{Ecopedagogía o Educación Ambiental}

Entre las ciencias de la naturaleza, destaca por su valor formativo la Educación Ambiental, que ha desembocado en una Ecoformación o Ecopedagogía. Como transversal, no puede ser un tema aislado en el currículum, sino un proceso conjunto, sistémico y organizado que implique a todas las disciplinas. Se reconoce su importancia en el presente.

Mención especial merece la educación ambiental. Una cultura ecológica preparará a los ciudadanos para comprender los problemas de la Tierra, creando un talante de respeto y de mejora de las riquezas comunes; contribuirá a la práctica de un espíritu comunitario y cósmico. Los habitantes de la Tierra tendrán conciencia de pertenecer a una misma casa a la que hay que cuidar y transformar (Rodríguez Rojo, 1996, 107).

El papel de la lengua en este tema es el mismo que en el aprendizaje de las ciencias en general. Para celebrar los días mundiales del medio ambiente ( 5 de junio), del agua ( 2 de octubre), de la biodiversidad ( 29 de diciembre)... se plantean proyectos transversales donde deberán leerse textos, ver películas, redactar conclusiones. Es recomendable el movimiento de Escuelas Verdes, pero todas pueden realizar, mediante asambleas en que debatirán la aprobación de su Agenda 21 o de la misma Carta de la Tierra.

Entre otros textos conocidos se puede trabajar el Mensaje del Gran Jefe Seattle, de gran valor educativo, ecológico y literario, aunque no hay ninguna prueba de que fuera pronunciado realmente por el Jefe:

[...] « Enseñad a vuestros hijos lo que nosotros hemos enseñado a nuestros hijos: la tierra es nuestra madre. Lo que afecte a la tierra, afectará también a los hijos de la tierra. Si los hombres escupen a la tierra, se escupen a sí mismos. Porque nosotros sabemos esto: la tierra no pertenece al hombre, sino el hombre a la tierra. Todo está relacionado como la sangre que une a una familia. El hombre no creó el tejido de la vida, sino que simplemente es una fibra de él. Lo que hagáis a ese tejido, os lo hacéis a vosotros mismos. 
Es importante usar críticamente los medios de comunicación como fuentes de información. Para concienciar de esta problemática se deberá leer, debatir y elaborar propuestas.

\subsubsection{Educación para la Salud}

Por una parte se vincula al conocimiento científico y por otra a los valores vitales. El respeto y la conservación del entorno empiezan por el respeto a uno mismo, al cuerpo y su salud, lo que implica al bienestar. Planteamos definir salud, comparando y debatiendo conceptos (Cid, Dapía, Trías y Payá, 2001, 180-183):

«Estado de completo bienestar físico, mental y social y no solo ausencia de enfermedad y minusvalía.» OMS, 1946).

«Capacidad de desarrollar el potencial personal y responder de forma positiva a los retos del ambiente.» (OMS, 1985).

Salud es una manera de vivir autónoma, solidaria, alegre, armoniosa y en equilibrio funcional, físico y psíquico, de la persona integrada en su medio natural y social. El debate girará en torno a las características positivas de este estado, incluyendo la alegría de la vida, la aceptación realista de uno mismo y el esfuerzo por transformar la realidad cotidiana con responsabilidad social.

Comentar textos como el Juramento hipocrático, algún poema o fragmentos de obras literarias que traten de la salud física y mental:

El último estornudo de un pesimista (Mario Benedetti)

Otorrinolaringología (García Márquez)

La montaña mágica (Thomas Mann)

Diario del año de la peste (Daniel Defoe)

El amor en los tiempos de cólera (García Márquez)

Los textos útiles son numerosos. La obra satírica de Rabelais pone de manifiesto excesos en la alimentación. El Quijote es el mejor para hablar de salud mental. Y también contiene ideas valiosas:

«Come poco y cena más poco; que la salud de todo el cuerpo se fragua en la oficina del estómago.» «No comas ajos ni cebollas, porque no saquen por el olor tu villanería.»

Se pueden aprender refranes sobre la salud, la alimentación sana y los remedios naturales. En inglés dicen que una manzana al día mantiene lejos al médico (An apple a day keeps the doctor away). Trabajando textos como recetas de cocina sanas, en la prevención del consumo de substancias tóxicas, obesidad, bulimia o anorexia, se puede usar la lengua en campañas de sensibilización. En el Día Mundial de la Salud (7 de abril) se programan sesiones de cine-fórum de películas con sensibilidad: Cuarta planta, Diario de Noa, Big Fish, Séptimo sello y otras sobre epidemias. En situaciones de pandemia como la de 2020, nos va la vida en ello, aprenderemos responsabilidad por el convencimiento a través del diálogo y debate. Y no olvidemos que los valores vitales suponen el sentido del humor. Aprender a contar chistes, forma parte de la risoterapia. 


\subsection{Ciencias Sociales}

El discurso propio de las ciencias sociales puede partir de las competencias lingüísticas (descripción, explicación, justificación, interpretación y argumentación), para dotar de sentido a la información y construir el conocimiento social. Intervienen diferentes tipos de lenguajes (icónicos, simbólicos, cartográficos, audiovisuales, informáticos, etc.) para comprender e interpretar la realidad. Como en otras áreas, el vocabulario propio permite la construcción de un discurso científico, preciso y riguroso. Se mejoran las habilidades comunicativas en situaciones de interacción oral como son los debates.

Imágenes y mapas aparte, las ciencias sociales necesitan usar constantemente la lengua. Por su papel en las competencias metodológicas, la lengua interviene en la comprensión de los procesos de convivencia. De manera especial, la competencia plurilingüe e intercultural contribuye a la competencia social y ciudadana, entendida como habilidades y destrezas para la convivencia, el respeto y entendimiento entre las personas. Aprender lengua es, ante todo, comunicarse con otros, tener contacto con distintas realidades y valorar la propia expresión como muestra de apertura a los demás.

\subsubsection{Geografia}

Además de textos descriptivos de paisajes y de viajes, el significado de la toponimia proporciona motivos para aprender el lenguaje. El estudio del medio, homegeography, es uno de los mejores ejemplos de proyecto interdisciplinario. En él, la intervención del área lingüística es constante, siempre relacionada con la vida real. Para conocer detalles del entorno, se realizan encuestas a los vecinos, a la población, se invita a personas mayores para dar conferencias, se escriben los resultados de las indagaciones. Junto al mapa, se acompañan textos de alumnos. Del entorno próximo se pasa a zonas más lejanas, para lo cual se necesita la prensa, televisión o Internet.

Uno de los principales medios de aprender es la excursión y en ella se conversa, se recoge material, que luego se transcribe y expone. A los reportajes fotográficos hay que añadirles un texto. También el cine y la televisión son motivos constantes para admirar regiones lejanas. La novela de viajes es como una excursión literaria.

Se describe (adjetivos), se clasifican hechos físicos y humanos (terminología), se busca información y se lee de manera comprensiva, se escriben síntesis de cada comarca o región, habitualmente en equipo, para lo cual deberán conversar. También se realizan exposiciones orales públicas con apoyo de mapas, murales o diapositivas.

\subsubsection{Historia}

En esta área, casi todo el aprendizaje se basa en lo verbal. Se investiga con documentos e inscripciones; sus resultados se exponen en forma de textos narrativos y explicativos. Hoy, más que la memoria, es importante la comprensión, con lo que predomina la abstracción y se desplazan términos como imperio, súbditos, fábrica... por desarrollo, cambio, causa, efecto... Se substituye una historia de reyes y héroes por otra del pueblo como protagonista. Para 
entenderlo, proponemos el comentario del poema Preguntas de un obrero ante un libro, de Bertolt Brecht:

«Tebas, la de las Siete Puertas, ¿quién la construyó?

En los libros figuran los nombres de los reyes.

¿Arrastraron los reyes los grandes bloques de piedra?

$\ldots$

El joven Alejandro conquistó la India.

¿Él solo?

César venció a los galos.

¿No llevaba consigo ni siquiera un cocinero?

Felipe II lloró al hundirse

su flota. ¿No lloró nadie más?

...

Una victoria en cada página.

¿Quién cocinaba los banquetes de la victoria?

Un gran hombre cada diez años.

¿Quién pagaba sus gastos?

Una pregunta para cada historia.»

En literatura, como en arte, la caracterización de cada movimiento artístico (renacimiento, barroco, romanticismo, modernismo...) requiere una contextualización histórica a la que puede ayudar la biografía de los autores, enmarcados en su época.

Otros recursos son conversaciones durante excursiones o paseos por museos, monumentos o lugares históricos, juegos cooperativos, simulaciones, teatro, dramatización y juego de rol. Y el cine con base histórica.

\subsubsection{Educación para la Ciudadanía y para la Paz}

Se necesita una práctica sistemática del debate y todo lo que implica (saber escuchar, exponer y argumentar), así como de la gestión de diversas fuentes de información para la construcción del conocimiento. Los mensajes, explícitos e implícitos, de estas fuentes han de ser valorados críticamente, especialmente los de la publicidad y los medios de comunicación. También atañe a la lengua la exposición oral y escrita de resultados en forma de sentimientos, ideas y opiniones, participando en debates donde se compartan opiniones argumentadas y juicios de forma asertiva. Se puede proponer la simulación de una sesión del parlamento o ayuntamiento. El lenguaje sirve para entendernos (comprensión interpersonal, resolución de conflictos, mediación). La palabra 'parlamento' viene de parabolare, hablar. Hablando la gente se entiende.

Definir lo que es paz, ni ausencia de guerra ni período entre dos guerras. Definir también violencia, conflicto, guerra. Debatir y proponer soluciones positivas a los conflictos.

Otras actividades: el Día Internacional de la no Violencia y la Paz, DENIP, 30 de enero, se puede celebrar con películas, poemas, canciones. Entre los innumerables textos posibles, sugerimos a Confucio, Tagore, Tolstoi (Guerra y paz), Luther King, Amos Oz, Berta de Suttner (Abajo las armas)... 
El uso de un lenguaje inclusivo, no solo en las formas, sino en la misma raíz, contribuirá a una educación por la igualdad superando el injusto techo de cristal de la mujer.

\subsection{Educación Artística. Lenguaje musical y plástico}

Las lenguas son un elemento cultural básico para el arte. La lectura, comprensión y valoración de las obras literarias participa en el desarrollo de la competencia artística y cultural. La creación de textos tiene relación con otras manifestaciones artísticas: música, dibujo, pintura. Dado un poema o un cuento, se plantea ilustrarlo o, al revés, de un cuadro se puede escribir una descripción o una poesía. Serrat, Paco Ibáñez o Amancio Prada han puesto música a poemas famosos. La musicalidad ya estaba en los versos.

La comunicación no verbal, en el teatro, cine, cómic, lenguaje cinematográfico o audiovisual, propicia una lectura de la imagen que la verbaliza mostrando la transversalidad de sus lenguajes. En música, la más universal de las artes, se cantan canciones, actividad muy motivadora: trabajaremos las letras, su significado, métrica, figuras literarias, contenido, autor si cabe. Combinaremos canciones populares, modernas y en otras lenguas, canciones del mundo.

\section{CONCLUSIÓN}

Se ha intentado mostrar como con todas las asignaturas se practican las competencias comunicativas, se pueden aprender y mejorar en áreas no lingüísticas. Todavía es posible ir más allá con una programación y un trabajo interdisciplinario. Las mismas clases de lengua y literatura o de idioma moderno han de compartir los valores de la transversalidad, que son los de presentar una forma más coherente y enlazada del conocimiento humano. Un uso exigente, pulcro y elegante del lenguaje en todas las materias confiere un extraordinario valor añadido al aprendizaje y desarrolla el pensamiento y la expresión.

\section{REFERENCIAS BIBLIOGRÁFICAS}

Armstrong, T. (2006). Inteligencias múltiples en el aula. Guía práctica para educadores. Barcelona: Paidós, $2^{\mathrm{a}}$ impresión, 2018.

Brecht, B. (1968). Poemas y canciones. Madrid: Alianza.

Ceccarelli, L. (1977). Didáctica de la observación científica. Barcelona: Fontanella.

Cid, X. M., Dapía, M. D., Trías, P. y Payá, M. (2001). Valores transversales en la práctica educativa. Madrid: Síntesis.

Darwich, M. (2003). A Rhyme for the Odes, 91, Unfortunately, It Was Paradise, Regents of the University of California.

Enkvist, I. (2011). La buena y la mala educación. Ejemplos internacionales. Madrid: Encuentro. González Lucini, F. (1994). Temas transversales y áreas curriculares. Madrid: Anaya.

Josa, L. (2020). Por qué leer a los clásicos nos hace más inteligentes. Recuperado de https:// theconversation.com/por-que-leer-a-los-clasicos-nos-hace-mas-inteligentes-129691 [Consulta: 30-08-2020]. 
Lemke, J. L. (1997). Aprender a hablar ciencia. Lenguaje, aprendizaje y valores. Barcelona: Paidós.

Lladó, J. y Llobera, M. (1999). Reflexions i propostes per al tractament de les llengües en els centres d'ensenyament. Palma de Mallorca: Moll; COFUC.

Lomas, C., Osoro, A. y Tusón, A. (1993). Ciencias del lenguaje, competencia comunicativa y enseñanza de la lengua. Barcelona: Paidós-Mec.

Mallart, A. (2009). Enseñar matemáticas con conciencia transdisciplinar. En S. de la Torre y M. A. Pujol (Eds.), Educar con otra conciencia. Una mirada ecoformadora y creativa de la enseñanza (pp. 215-224). Barcelona: Davinci.

Mallart, J. (2007). Ecoformación, más allá de la educación ambiental. En S. de la Torre, M. A. Pujol y G. Sanz, Transdisciplinariedad y ecoformación. Una nueva mirada sobre la educación (pp. 151-166). Madrid: Universitas.

Mallart, J. (2002). Competencias básicas y enseñanzas mínimas del currículum de lengua. Revista de Educación, (329), 219-238.

Mallart, J. (2011). Competències educatives. Revisió conceptual, cronològica i bibliogràfica. Revista Catalana de Pedagogia, (7), 249-282.

Mallart, J. y Sarramona, J. (Coords.) (2013). Competències bàsiques de l'àmbit lingüístic. Llengua i literatura (primària i secundària obligatòria). Barcelona: Departament d'Ensenyament. Nueva edición 2015. Recuperado de http://ensenyament.gencat.cat/web/. content/home/departament/publicacions/colleccions/competencies-basiques/eso/ eso-linguistic-ca-es-literatura.pdf [Consulta 30/03/2018].

Manea, N. (2008). La llengua nòmada. Barcelona: Arcàdia.

Niss, M. (1999). Competencies and Subject Description. Uddanneise, (9), 21-29.

Palos, J. (1998). Educar para el futuro: temas transversales del currículum. Bilbao: Desclée de Brouwer.

Rodríguez Rojo, M. (1995). La Educación para la Paz y el interculturalismo como tema transversal. Barcelona: Oikos-Tau.

Rodríguez Rojo, M. (1996). Transversalidad y democracia en el contexto europeo. Revista de Educación, (309), 95-126.

Rondal, J. (1980). Lenguaje y educación. Barcelona: Médica y Técnica.

Rosales, C. (1984). Didáctica de la comunicación verbal. Madrid: Narcea.

Rosales, C. (2006). Perspectivas de Educación para la Salud. Santiago: Tórculo.

Rosales, C. (2010). La planificación de la enseñanza por competencias: ¿Qué tipo de innovación implica? Innovación educativa, (20), 77-88. Recuperado de http://hdl.handle. net/10347/4999

Rosales, C. (2015). Evolución y desarrollo actual de los Temas Transversales: posibilidades y límites. Foro de Educación, 13(18), 143-160. DOI: https://doi.org/10.14516/ fde.2015.013.018.008

Rosales, C. (2019). ¿Cuáles son los ámbitos de transversalidad educativa? Innovación educativa, (29), 109-123. DOI: https://doi.org/10.15304/ie.29.6023

Salinas, P. (1967). El defensor. Madrid: Alianza. 
Salvador, F. y Camacho, S. (1991). El área de lengua en el currículo escolar. Sugerencias para el diseño curricular. Educadores, 157, 95-113.

Scurati, G. y Damiano, E. (1977). Interdisciplinariedad y didáctica. A Coruña: Adara.

Seattle (1994). Nosaltres som una part de la Terra. Missatge del Gran Cabdill Seattle. Barcelona: Hesperus.

Unamuno, M. de (1968). Rosario de sonetos líricos. En Poesía completa. Madrid: Alianza.

Yus, R. (1996). Temas transversales: Hacia una nueva escuela. Barcelona: Graó. 